Published in final edited form as:

J Dent Res. 2007 April ; 86(4): 373-377.

\title{
Tobacco use and incidence of tooth loss among US male health professionals
}

\author{
T. Dietrich ${ }^{1,2}$, N. N. Maserejian ${ }^{3,4}$, K. J. Joshipura ${ }^{5}$, E.A. Krall ${ }^{1}$, and R. I. Garcia ${ }^{1,6}$ \\ 1 Dept of Health Policy \& Health Services Research, Boston University Goldman School of Dental Medicine \\ 2 Dept of Periodontology and Oral Biology, Boston University Goldman School of Dental Medicine \\ 3 Dept of Epidemiology, Harvard School of Public Health \\ 4 New England Research Institutes \\ 5 University of Puerto Rico, Division of Dental Public Health, School of Dentistry, Medical Science Campus \\ 6 VA Normative Aging Study, VA Boston Healthcare System
}

\begin{abstract}
Data on the dose dependent effect of smoking and smoking cessation on tooth loss are scarce. We hypothesized that smoking has a dose and time dependent effect on tooth loss incidence. We used longitudinal data on tobacco use and incident tooth loss in 43,112 male health professionals between 1986 and 2002. In multivariate Cox models, current smokers of 5 to 14 and $45+$ cigarettes daily had a two-fold (HR: 1.94; 95\% CI: 1.72, 2.18) and three-fold (HR: 3.05; 95\% CI: 2.38, 3.90) higher risk of tooth loss, respectively, compared to never-smokers. Risk decreased with increasing time since cessation, but remained elevated by $20 \%$ (95\% CI: $16 \%, 25 \%)$ for men who had quit $10+$ years before. Current pipe/cigar smokers had a $20 \%$ (95\% CI: 1.11, 1.30) increased risk of tooth loss compared to never and former smokers of pipes/cigars.
\end{abstract}

\section{Keywords}

Periodontitis; Smoking; Tobacco; Tooth loss

\section{INTRODUCTION}

Cigarette smoking has emerged as the single most important modifiable behavioral risk factor for periodontal disease (Hujoel et al., 2003; Palmer et al., 2005; Tomar and Asma, 2000). Cigarette smoking has also been associated with higher prevalence of edentulousness and fewer remaining teeth in cross-sectional studies (Axelsson et al., 1998; Daniell, 1983; Gilbert $e t$ al., 1993; Krall et al., 1997; Linden and Mullally, 1994), and with increased rates of tooth loss in longitudinal studies (Ahlqwist et al., 1989; Eklund and Burt, 1994; Holm, 1994; Jansson and Lavstedt, 2002; Krall et al., 1997). However, many previous studies were relatively small or used broad categorizations of smoking history. Therefore, the dose-response relationship between cigarette smoking and tooth loss is not well characterized. Furthermore, little is known about the time-dependent effect of smoking cessation on risk of tooth loss. In a study of 1,031 Swedish women, risk of tooth loss over 12 years was similar between never smokers and former smokers who had quit any time before baseline (Ahlqwist et al., 1989). Krall et al. found an

Corresponding author: Thomas Dietrich, DMD, MD, MPH Dept of Health Policy \& Health Services Research Boston University School of Dental Medicine 715 Albany St, 560, $3^{\text {rd }}$ floor Boston, MA 02118 USA T: +1-617-414-1130 F: +1-617-638-6381 tdietric@bu.edu. 
intermediate rate of tooth loss for male smokers who quit at any time during the 3 to 26 years of follow-up and concluded that it may take decades for quitters to return to the tooth loss rate of never-smokers (Krall et al., 1997). The use of other types of tobacco, such as cigar, pipe, and smokeless tobacco, is also likely to be related to tooth loss risk, but few studies have explored this hypothesis. A cross-sectional analysis of 705 subjects of the Baltimore Longitudinal Study of Aging found that the number of missing teeth was higher among smokers of pipes and cigars (Copeland et al., 2004). Both pipe and cigar smoking were independently associated with increased tooth loss risk in a longitudinal study of 690 men (Krall et al., 1999). In a representative survey of the US population (NHANES III), history of smokeless tobacco use was associated with periodontal disease prevalence (Fisher et al., 2005). However, whether or not the use of smokeless tobacco increases the risk of tooth loss has not been investigated.

The purpose of the present study was to evaluate the associations between the various forms of tobacco use (cigarette smoking, pipe or cigar smoking, chewing tobacco) and smoking cessation and the incidence of tooth loss in a large, prospective study of U.S. health professionals.

\section{MATERIALS AND METHODS}

The Health Professionals' Follow-Up Study (HPFS) is an ongoing longitudinal cohort study of 51,529 male health professionals, including dentists (58\%), veterinarians (20\%), pharmacists (8\%), optometrists (7\%), osteopaths (4\%), and podiatrists (3\%). Participants were 40 to 75 years of age at the study baseline in 1986. Participants completed mailed questionnaires every 2 years to provide information on medical history, and health behaviors. On average, over $90 \%$ of the baseline population responded to each follow-up questionnaire. This study was approved by the Human Subjects Committee at Harvard School of Public Health.

For the present analysis, we excluded men if at baseline they had: missing data for cigarette smoking status, a cancer diagnosis by 1986, self-reported daily caloric intake outside the plausible range of 800 to 4200 , or 70 of the 131 dietary questions left blank. We also excluded 742 edentulous men (29\% never, $57 \%$ former and $15 \%$ current cigarette smokers). The final analytic sample included 43,112 men who contributed 569,366 person-years.

\section{Exposure Assessment}

The baseline HPFS questionnaire asked detailed questions on the history of cigarette smoking. Men who had smoked less than 20 packs of cigarettes in their lifetime were defined as never smokers. Ever smokers of cigarettes reported the average number of cigarettes per day (0-4, $5-14,15-24,25-34,35-44,45+\mathrm{cig} / \mathrm{d})$ and, if former smokers, time since cessation ( $<1$ year, 1-2 years, 3-5 years, 6-9 years, 10+ years). Information on cigarette and cigar or pipe smoking (current, yes/no) was updated biennially. Ever-use of chewing tobacco was ascertained in the 1996 questionnaire ("Have you ever chewed tobacco at least once a week for a year?").

\section{Outcome Assessment}

Participants reported baseline number of teeth in 1986 and incident tooth loss in two-years intervals thereafter. Missing values on incident tooth loss were assumed to represent no tooth loss during that follow-up period because only $\approx 10 \%$ of participants experienced tooth loss biennially. Self-reported number of teeth and tooth loss have been found to be highly accurate in various populations (Douglass et al., 1991; Gilbert et al., 2002; Pitiphat et al., 2002). Thus, in this population of dentists and health professionals, self-reported number of teeth and tooth loss are likely to have high validity. 


\section{Assessment of potential confounders}

Diet was assessed at baseline and every four years thereafter with an expanded semiquantitative food frequency questionnaire (Willett $e t$ al., 1985). Every two years, questionnaires also assessed use of multivitamins and specific vitamin supplements. Validity of the dietary data has been documented by comparisons with multiple weighed dietary records (Rimm et al., 1992). Physical activity was assessed biennially and calculated as the sum of the activityspecific metabolic equivalent (MET) hours/week as a measure of total leisure-time physical activity.

\section{Data Analysis}

Person-time for each participant was calculated from the date of return of the 1986 questionnaire to the date of first incident tooth loss, death, or January 31, 2002, whichever occurred first.

Cox proportional-hazards models were used to obtain hazard ratios and $95 \%$ confidence intervals for the association between tobacco use and risk of tooth loss. Former smokers were categorized according to time since cessation $(<1,1-2,3-5,6-9$ and 10+ years ago), and current smokers were categorized according to intensity $(<5,5-14,15-24,25-34,35-44,45+$ cigarettes/ day).

All models contained cigarette, cigar/pipe and chewing tobacco variables, to mutually adjust for type of tobacco use. The basic model included age (months) and race (Caucasian vs. other). A multivariable model further adjusted for other variables that were significantly associated with tooth loss $(\mathrm{p}<0.05)$ in bivariate analyses or that changed the effect estimates of the tobacco use/tooth loss association by $\geq 5 \%$. Cigarette smoking, pipe/cigar smoking, body mass index (BMI), physical activity, diabetes, use of multivitamins/vitamin supplements and intakes of nutrients/food groups were modeled as time-dependent variables.

Sensitivity analyses restricted the cohort to dentists, because reasons for tooth loss and accuracy of self-reports may differ from non-dentists. Finally, because smoking increases the risk of various cancers and cancer treatment (i.e., radiotherapy and chemotherapy) that may be associated with increased tooth loss, a separate analysis censored person-time at the follow-up preceding a first report of a cancer diagnosis.

All statistical tests were two-sided and calculated using SAS 9.1.2 (SAS Institute Inc., Cary, NC).

\section{RESULTS}

At baseline in 1986, 3,765 (8.7\%) men were current cigarette smokers and 18,950 (44.0\%) were former cigarette smokers. Age, race, BMI, profession, multivitamin use and total caloric intake were similar among never, former and current smokers (Table 1). Compared to never smokers, current smokers, and particularly current heavy smokers, were more likely to be diabetic and physically inactive, less likely to have had a routine physical exam, less likely to take vitamin C supplements and more likely to consume alcohol. Furthermore, smokers had lost more teeth prior to baseline.

Over the course of the follow-up between 1986 and 2002, 13,656 men lost at least one tooth (average time until tooth loss: 8.4 years, range $0.1-13.4$ years). There was a strong, dosedependent association between cigarette smoking and risk of tooth loss (Table 2). Among current cigarette smokers, the risk of tooth loss increased steadily with increasing smoking intensity. Compared to never smokers, current smokers who smoked 5-14 cigarettes per day had twice the risk of tooth loss (HR: 1.94; 95\% CI: 1.72, 2.18), and current smokers of 45 or 
more cigarettes per day had a three-fold higher risk of tooth loss (HR: 3.05; 95\% CI: 2.38 , 3.90). The association was independent of other tobacco use, age, race, BMI, physical activity, diabetes, profession (dentist vs. non-dentist), routine medical exam, alcohol consumption, caloric intake, multivitamin use, vitamin $\mathrm{C}$ supplement use.

Among former cigarette smokers, risk of tooth loss decreased steadily with increasing time since cessation (Table 2). Compared to never smokers, men who quit smoking 10 or more years ago had 20\% (95\% CI: 16\%, 25\%) greater risk of tooth loss. Multivariable adjustments and restriction of the analysis to dentists yielded very similar hazard ratio estimates for cigarette smoking.

Current pipe or cigar smoking was associated with a 20\% (multivariate HR:1.20; 95\% CI: 1.11, 1.30) increased risk of tooth loss compared to never or former smokers of pipes or cigars.

Results were similar when the analysis was restricted to dentists. Ever-use of chewing tobacco was associated with incident tooth loss in the age- and race-adjusted analysis (HR:1.27; 95\% CI: 1.16, 1.39) as well as in the full multivariate model (HR:1.14; 95\% CI: 1.04, 1.24), although the association was attenuated when additional covariates were included. When restricted to dentists, however, no statistically significant association between chewing tobacco and tooth loss was observed (HR: 1.06; 95\% CI: 0.90, 1.26).

Censoring of person-time prior to a first diagnosis of cancer did not change the results (data not shown).

\section{DISCUSSION}

In this large, prospective cohort study of male health professionals, we found a strong, dosedependent association between cigarette smoking and the risk of tooth loss, independent of other risk factors and potential confounders. The risk of tooth loss declined after smoking cessation as a function of time since cessation; however, compared to never smokers, the risk of tooth loss remained elevated even 10 years after smoking cessation. Current smokers of pipe or cigars also had increased risk of tooth loss. Ever-use of chewing tobacco seemed to increase risk as well, though estimates were not as robust as for other types of tobacco.

Important strengths of this study are its prospective design, large sample size, long follow-up, and detailed, biennially updated data on smoking, allowing for fine exposure categories and precise estimates. Considering that tooth loss is the outcome of a complex process that may involve numerous factors that are also related to tobacco use (e.g., diet, health behaviors), another strength of this study is our ability to adequately control for such confounders. Furthermore, the cohort's relative homogeneity minimizes potential confounding by factors such as socio-economic status and access to care.

The path by which cigarette smoking affects tooth loss is presumed to involve periodontitis. In addition to periodontitis, dental caries may also contribute to the increased risk of tooth loss among smokers (Ylostalo et al., 2004). Associations have been reported between cigarette smoking and root caries (Fure, 2004; Hahn et al., 1999; Phelan et al., 2004), coronal caries (Axelsson et al., 1998; Drake et al., 1997), endodontic treatment (Krall et al., 2006a) and periapical periodontitis (Kirkevang and Wenzel, 2003), although not consistently (Bergstrom et al., 2004).

We found that risk of tooth loss declines as early as one year after smoking cessation. However, it may take more than 10 years of abstinence for the risk to decline to that of never smokers. This is in agreement with results from the VA Dental Longitudinal Study, where after 12 years of cessation the risk of tooth loss approached that of never smokers (Krall et al., 1997; Krall et al., 2006b). 
These results suggest that following smoking cessation, the effect of smoking on tooth loss declines less rapidly than the effect of smoking on periodontitis (Bergstrom et al., 2000; Bolin et al., 1993; Tomar and Asma, 2000). Using NHANES III data, we estimated the half-life of the effect of smoking on periodontal disease at 1.5 years (Dietrich and Hoffmann, 2004), suggesting that the risk for periodontal disease for former smokers should approach that of never smokers approximately 6 years after quitting. This apparent difference in the half-life of the effects of smoking on periodontal disease vs. tooth loss may be explained by factors other than periodontitis, such as caries, that also mediate the effect of smoking on tooth loss risk.

Our finding that cigar or pipe smoking is associated with risk of tooth loss confirms earlier work. In a cross-sectional study, men who smoked pipes or cigars had a higher prevalence of moderate or severe periodontitis and fewer teeth remaining than non-smokers (Albandar $e t$ $a l ., 2000)$. In a longitudinal study, we earlier found that cigar smoking is associated with significantly higher rates of tooth loss (Krall et al., 1999). However, it is uncertain by exactly how much the risk increases as we only had a dichotomous measure of current cigar or pipe smoking, i.e., the comparison group included both never and former smokers.

We found a significant positive association between ever-use of chewing tobacco and risk of tooth loss only among the non-dentist health professionals. Previous studies of smokeless tobacco and tooth loss are lacking, and our results should be interpreted with caution. Multivariable adjustment resulted in a marked attenuation of the hazard ratio, and no association was evident when the analysis was restricted to dentists. Although residual or unknown confounding may explain the small association found, it is likely that our measure of chewing tobacco use was insufficient to accurately estimate an association with tooth loss. We did not have specific information on dose, duration, or timing (i.e., ages) of chewing tobacco use, and it may be that a large proportion of everusers were men who used chewing tobacco in the distant past.

Our results provide evidence for a strong time- and dose-dependent association between tobacco smoking and risk of tooth loss among men. However, these results may not be directly generalizable to women. A somewhat stronger association between smoking and tooth loss was reported among women in a cross-sectional study of 8,409 young Finnish adults (Ylostalo et al., 2004). Lastly, as the majority of HPFS participants were Caucasian, generalizability of our findings to other racial/ethnic groups is uncertain.

In conclusion, there is a strong association between cigarette, pipe, or cigar smoking and subsequent tooth loss in men. Current heavy cigarette smokers have a three-fold greater risk of incident tooth loss, compared to never smokers. The risk declines soon after cessation of cigarette smoking, but remains elevated for more than ten years compared to never smokers.

\section{ACKNOWLEDGEMENTS}

This investigation was supported by NIDCR Grants R03 DE016357 and K24 DE00419, and the U.S. Dept. of Veterans Affairs.

\section{REFERENCES}

Ahlqwist M, Bengtsson C, Hollender L, Lapidus L, Osterberg T. Smoking habits and tooth loss in Swedish women. Community Dent Oral Epidemiol 1989;17(3):144-7. [PubMed: 2786792]

Albandar JM, Streckfus CF, Adesanya MR, Winn DM. Cigar, pipe, and cigarette smoking as risk factors for periodontal disease and tooth loss. J Periodontol 2000;71(12):1874-81. [PubMed: 11156044]

Axelsson P, Paulander J, Lindhe J. Relationship between smoking and dental status in 35-, 50-, 65-, and 75-year-old individuals. J Clin Periodontol 1998;25(4):297-305. [PubMed: 9565280]

$J$ Dent Res. Author manuscript; available in PMC 2008 November 11. 
Bergstrom J, Eliasson S, Dock J. A 10-year prospective study of tobacco smoking and periodontal health. J Periodontol 2000;71(8):1338-47. [PubMed: 10972650]

Bergstrom J, Babcan J, Eliasson S. Tobacco smoking and dental periapical condition. Eur J Oral Sci 2004;112(2):115-20. [PubMed: 15056107]

Bolin A, Eklund G, Frithiof L, Lavstedt S. The effect of changed smoking habits on marginal alveolar bone loss. A longitudinal study. Swed Dent J 1993;17(5):211-6. [PubMed: 8291030]

Copeland LB, Krall EA, Brown LJ, Garcia RI, Streckfus CF. Predictors of tooth loss in two US adult populations. J Public Health Dent 2004;64(1):31-37. [PubMed: 15078059]

Daniell HW. Postmenopausal tooth loss. Contributions to edentulism by osteoporosis and cigarette smoking. Arch Intern Med 1983;143(9):1678-82. [PubMed: 6615088]

Dietrich T, Hoffmann K. A comprehensive index for the modeling of smoking history in periodontal research. J Dent Res 2004;83(11):859-63. [PubMed: 15505236]

Douglass CW, Berlin J, Tennstedt S. The validity of self-reported oral health status in the elderly. J Public Health Dent 1991;51(4):220-2. [PubMed: 1941773]

Drake CW, Beck JD, Lawrence HP, Koch GG. Three-year coronal caries incidence and risk factors in North Carolina elderly. Caries Res 1997;31(1):1-7. [PubMed: 8955986]

Eklund SA, Burt BA. Risk factors for total tooth loss in the United States; longitudinal analysis of national data. J Public Health Dent 1994;54(1):5-14. [PubMed: 8164192]

Fisher MA, Taylor GW, Tilashalski KR. Smokeless Tobacco and Severe Active Periodontal Disease, NHANES III. J Dent Res 2005;84(8):705-10. [PubMed: 16040726]

Fure S. Ten-year cross-sectional and incidence study of coronal and root caries and some related factors in elderly Swedish individuals. Gerodontology 2004;21(3):130-40. [PubMed: 15369015]

Gilbert GH, Duncan RP, Crandall LA, Heft MW, Ringelberg ML. Attitudinal and behavioral characteristics of older Floridians with tooth loss. Community Dent Oral Epidemiol 1993;21(6):3849. [PubMed: 8306618]

Gilbert GH, Chavers LS, Shelton BJ. Comparison of two methods of estimating 48-month tooth loss incidence. J Public Health Dent 2002;62(3):163-9. [PubMed: 12180044]

Hahn P, Reinhardt D, Schaller HG, Hellwig E. Root lesions in a group of 50-60 year-old Germans related to clinical and social factors. Clin Oral Investig 1999;3(4):168-74.

Holm G. Smoking as an additional risk for tooth loss. J Periodontol 1994;65(11):996-1001. [PubMed: 7853136]

Hujoel PP, del Aguila MA, DeRouen TA, Bergstrom J. A hidden periodontitis epidemic during the 20th century? Community Dent Oral Epidemiol 2003;31(1):1-6. [PubMed: 12542426]

Jansson L, Lavstedt S. Influence of smoking on marginal bone loss and tooth loss--a prospective study over 20 years. J Clin Periodontol 2002;29(8):750-6. [PubMed: 12390572]

Kirkevang LL, Wenzel A. Risk indicators for apical periodontitis. Community Dent Oral Epidemiol 2003;31(1):59-67. [PubMed: 12542433]

Krall EA, Dawson-Hughes B, Garvey AJ, Garcia RI. Smoking, smoking cessation, and tooth loss. J Dent Res 1997;76(10):1653-9. [PubMed: 9326897]

Krall EA, Garvey AJ, Garcia RI. Alveolar bone loss and tooth loss in male cigar and pipe smokers. J Am Dent Assoc 1999;130(1):57-64. [PubMed: 9919032]

Krall EA, Abreu Sosa C, Garcia C, Nunn ME, Caplan DJ, Garcia RI. Cigarette smoking increases the risk of root canal treatment. J Dent Res 2006a;85(4):313-7. [PubMed: 16567550]

Krall EA, Dietrich T, Nunn ME, Garcia RI. Risk of tooth loss after cigarette smoking cessation. Prev Chronic Dis 2006b;3(4):A115. [PubMed: 16978490]

Linden GJ, Mullally BH. Cigarette smoking and periodontal destruction in young adults. J Periodontol 1994;65(7):718-23. [PubMed: 7608851]

Palmer RM, Wilson RF, Hasan AS, Scott DA. Mechanisms of action of environmental factors--tobacco smoking. J Clin Periodontol 2005;32(Suppl 6):180-95. [PubMed: 16128837]

Phelan JA, Mulligan R, Nelson E, Brunelle J, Alves ME, Navazesh M, Greenspan D. Dental caries in HIV-seropositive women. J Dent Res 2004;83(11):869-73. [PubMed: 15505238]

Pitiphat W, Garcia RI, Douglass CW, Joshipura KJ. Validation of self-reported oral health measures. J Public Health Dent 2002;62(2):122-8. [PubMed: 11989207] 
Rimm EB, Giovannucci EL, Stampfer MJ, Colditz GA, Litin LB, Willett WC. Reproducibility and validity of an expanded self-administered semiquantitative food frequency questionnaire among male health professionals. Am J Epidemiol 1992;135(10):1114-26. [PubMed: 1632423]

Tomar SL, Asma S. Smoking-attributable periodontitis in the United States: findings from NHANES III. National Health and Nutrition Examination Survey. J Periodontol 2000;71(5):743-51. [PubMed: 10872955]

Willett WC, Sampson L, Stampfer MJ, Rosner B, Bain C, Witschi J, Hennekens CH, Speizer FE. Reproducibility and validity of a semiquantitative food frequency questionnaire. Am J Epidemiol 1985;122(1):51-65. [PubMed: 4014201]

Ylostalo P, Sakki T, Laitinen J, Jarvelin MR, Knuuttila M. The relation of tobacco smoking to tooth loss among young adults. Eur J Oral Sci 2004;112(2):121-6. [PubMed: 15056108] 


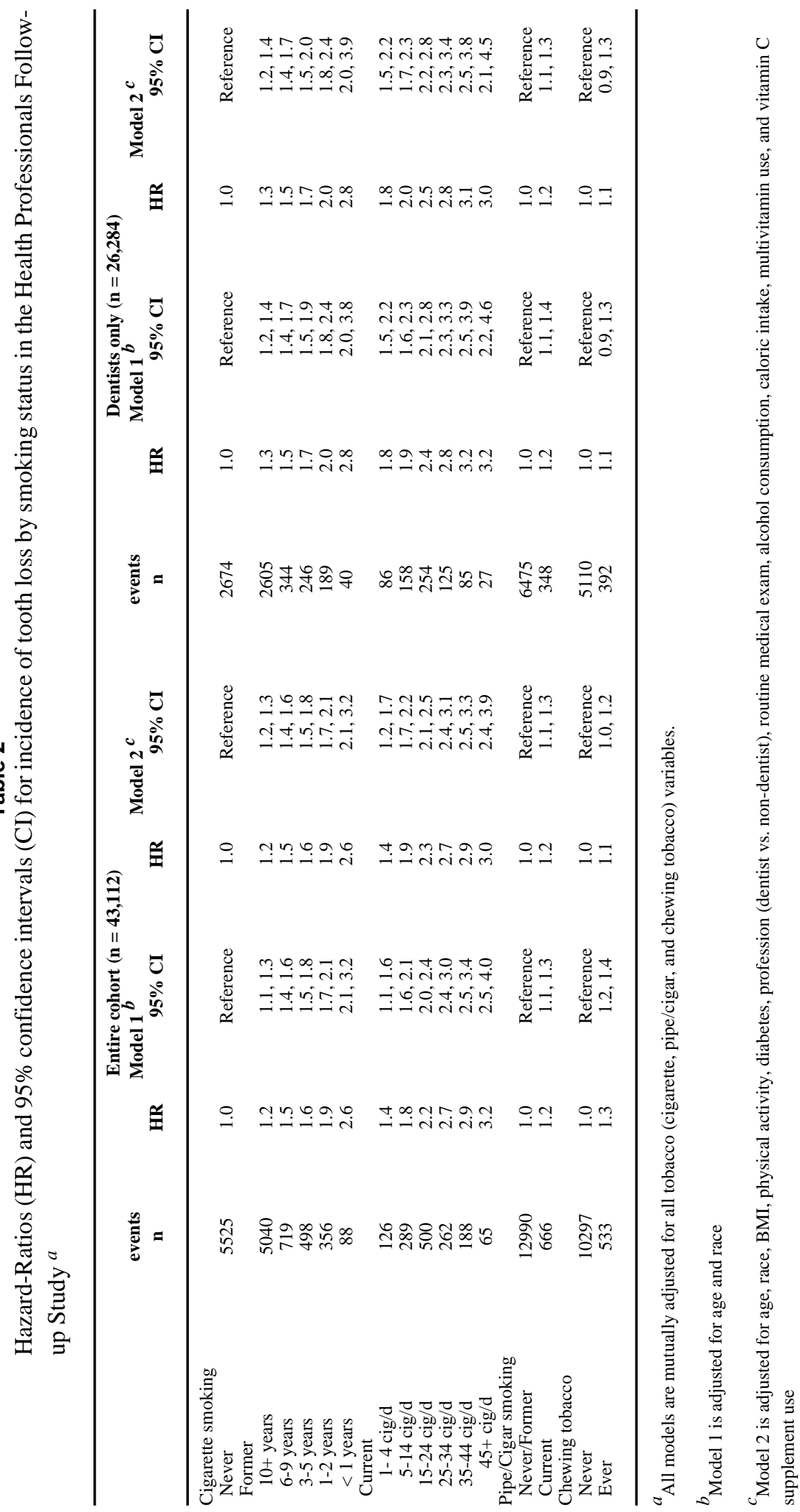

